\title{
The Influence of Spherical Nano-SiO 2 Content on the Thermal Protection Performance of Thermal Insulation Ablation Resistant Coated Fabrics
}

\author{
Guoyi Liu, ${ }^{1,2}$ Yuanjun Liu, ${ }^{1}$ and Xiaoming Zhao ${ }^{1}$ \\ ${ }^{1}$ School of Textiles, Tianjin Polytechnic University, Tianjin 300387, China \\ ${ }^{2}$ Tianjin Fire Protection Bureau, Tianjin 300090, China \\ Correspondence should be addressed to Xiaoming Zhao; texzhao@163.com
}

Received 2 April 2017; Revised 6 June 2017; Accepted 15 June 2017; Published 20 July 2017

Academic Editor: Jefferson Maul

Copyright (C) 2017 Guoyi Liu et al. This is an open access article distributed under the Creative Commons Attribution License, which permits unrestricted use, distribution, and reproduction in any medium, provided the original work is properly cited.

\begin{abstract}
In the high temperatures experienced in fire, radiant heat accounts for $80 \%$ of the total heat flow; therefore, improving the radiation protection is the best way to enhance the thermal protective performance of thermal insulation ablation resistant coated fabrics. To achieve this goal, the coating process and the ingredients used were optimized, spherical nano- $\mathrm{SiO}_{2}$ and other particles were added, and thermal insulation ablation resistant coated fabrics with high radiant heat reflectivity were prepared. The influence of the spherical nano- $\mathrm{SiO}_{2}$ content on the thermal protection performance of the prepared coated fabrics was investigated. Research showed that (1) the radiant heat reflectivity of the prepared coated fabrics improves significantly with increasing content of spherical nano- $\mathrm{SiO}_{2}$; when the mass fraction of spherical nano- $\mathrm{SiO}_{2}$ is $15 \%$, the reflectivity of coated fabrics is at its largest, and its average value was $74.30 \%$. At present, the average size of grains in samples increased 1.9 times; (2) after adding the spherical nano-SiO ${ }_{2}$, the thermal stability of the thermal insulation ablation resistant coated fabrics is significantly improved; the residual mass is as high as $88.49 \%$ at $1200^{\circ} \mathrm{C}$, which is $18.77 \%$ higher than the residual mass of the coated fabrics with no spherical nano-SiO $\mathrm{S}_{2}$ added at the same temperature.
\end{abstract}

\section{Introduction}

In the high temperature fire scene, very severe thermal degradation and carbonation of common coatings will occur; since the coated fabric system cannot withstand the ablation due to high temperature flame, it will be destroyed finally. Thermal insulation ablation resistant coated fabrics can tolerate the licking of high temperature flame in the short term, with no burning, no bubbles, and no peeling, and, at the same time, good heat insulation and mechanical properties are maintained [1-3]. Therefore, these types of fabrics offer excellent thermal protection in a high temperature fire environment, but there has been more focus on their ablation resistance and other aspects of thermal stability performance. At present, there are very few mechanisms and experimental studies when combining ablation resistance with other demands of fire protection $[4,5]$.

In the heat flow of high temperature fire, radiant heat accounts for more than $80 \%$, and the wavelength range is
$0.4-2$ microns, which is also called the heat ray [6-8]. Because the proportion of thermal radiation in the heat flow of high temperature fire is very large, emphasis needs to be aimed at the heat source when considering protection $[9,10]$.

A research done by $\mathrm{Zhu}$ et al. showed that the introduction of nano- $\mathrm{SiO}_{2}$ aerogel with phosphorus flame retardants had a significantly synergistic effect on improving the flame retardancy and inhibiting the release of smoke and toxic gases [11]. A study by Wang et al. reported that the prepared nanocomposite to which more nanosilicon dioxide was added had larger thermal storage capacity, better reliability and stability [12]. Nguyen et al. demonstrated that adding nanosilicon dioxide increased glass transition temperature and the impact strength of the prepared nanocomposite [13]. $\mathrm{Li}$ et al. proposed that the material with more nano- $\mathrm{SiO}_{2}$ content displayed an increased thermodynamic stability, an increased high temperature resistance, an increased tensile strength, and a decreased elongation at break [14]. Yang et al. found that silica nanoparticles based fresh water drilling 


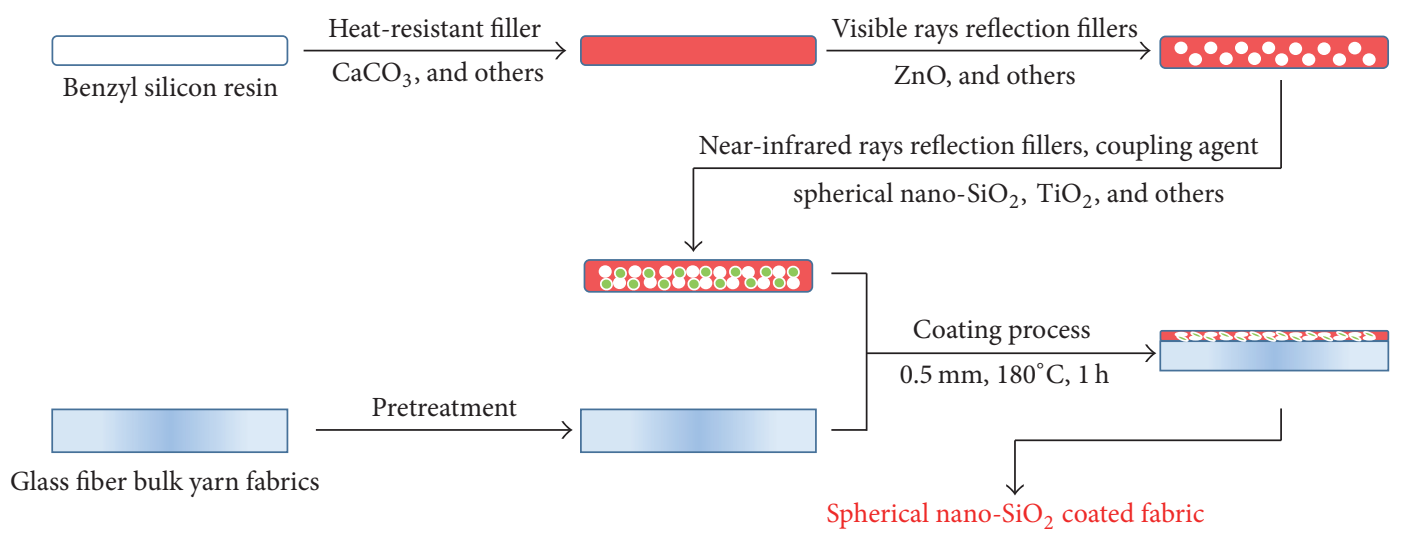

FIGURE 1: Flow diagram of spherical nano- $\mathrm{SiO}_{2}$ fabric coating process.

muds had good thermal stability up to $160^{\circ} \mathrm{C}$ [15]. Lee et al. reported that the proton conductivity as well as thermal stability was found to be higher for composite membranes which suggested that both properties are highly contingent on mesoporous $\mathrm{SiO}_{2}$ [16]. Loka et al. found that the prepared $\mathrm{SiO}_{2}$ deposited films showed a high infrared reflectance (72\%) which was recorded by using the UV-Vis-NIR spectrophotometer [17]. Krimmer et al. prepared a broadband multilayer dielectric mirror coating of $\mathrm{TiO}_{2} / \mathrm{SiO}_{2}$ which had a mean specular reflectivity approaching $98 \%$ over the wavelength range of $450-670 \mathrm{~nm}$ [18]. Fu et al. measured the spectral normal-hemispherical reflectance, transmittances, and normal emittances of silica aerogel and silica fiber samples and compared the two materials in the wavelengths of $0.38-15 \mu \mathrm{m}$ [19]. In conclusion, scholars have done a more in-depth theoretical analysis and experimental research of the $\mathrm{SiO}_{2}$ application in thermal protective performances, especially in infrared reflectivity.

In view of current researches and realistic fire protection requirements, nano- $\mathrm{SiO}_{2}$ and other selected nanoparticles were added to the coating system, and coated fabric composite materials with good reflection properties for thermal radiation and ablation resistance were prepared. In this article, the influence of the $\mathrm{SiO}_{2}$ particles on the reflectivity of thermal radiation for thermal insulation ablation resistant coated fabrics was investigated, the aim of which was to prolong the protection time of coated fabrics in the high temperature fire scene. Better and more effective methods are provided, and their protective mechanism is explained.

\section{Experiments}

\subsection{Materials and Reagents}

(1) Base Cloth. Glass fiber bulk yarn plain fabrics, gram weight $1000 \mathrm{~g} / \mathrm{m}^{2}$, provided by Zibo Sichuang Fiberglass Co., Ltd.

(2) Resin. Benzyl silicon resin, Type 1153, provided by the Shanghai Resin Factory.

(3) Functional Packing. Spherical nano- $\mathrm{SiO}_{2}$, provided by the Shanghai Naiou Nano Science Technology Co.,
Ltd.; rutile type $\mathrm{TiO}_{2}$, provided by Nanjing Tianxing New Materials Co., Ltd.; $\mathrm{ZnO}, \mathrm{Fe}_{2} \mathrm{O}_{3}, \mathrm{CaCO}_{3}$, and talcum powder were supplied as analytically pure reagents by Tianjin Guangfu Fine Chemical Industry Research Institute.

(4) Solvent and Coupling Agent. Anhydrous ethanol, analytically pure reagent, provided by the Tianjin Kemiou Chemical Reagent Co., Ltd. Coupling agent $\mathrm{KH} 550$, analytically pure reagent, provided by Tianjin Guangfu Fine Chemical Industry Research Institute.

2.2. Instruments. Ultraviolet visible (UV-Vis) near-infrared spectrophotometer, Type: Lambda 750, PerkinElmer Company, USA; field emission-scanning electron microscopy, Type: Gemini SEM500, ZEISS Company, Germany; X-ray diffractometer, Type: D8 DISCOVER, BRUKER Company, Germany; Thermogravimetric Analyzer, Type: STA 409 PC, NETZSCH Companies, Germany; X-ray photoelectron spectroscope, Type: K-alpha, ThermoFisher Company, USA; Ice characteristic X-ray spectrometer, Type: APOLLO XL, EDAX Company, USA were used.

2.3. The Preparation Process of Spherical Nano-SiO 2 Coated Fabrics. The preparation process of spherical nano- $\mathrm{SiO}_{2}$ coated fabrics, which was divided into the following three steps, is shown in Figure 1:

(1) Anhydrous ethanol was used to clean the base cloth, which was dried and put into the sample bag.

(2) Organic silicon resin was dissolved in anhydrous ethanol and fully stirred, before adding calcium carbonate, talcum powder, $\mathrm{Fe}_{2} \mathrm{O}_{3}$, and $\mathrm{ZnO}$. Mass fractions of $0 \%, 3 \%, 6 \%, 9 \%, 12 \%$, and $15 \%$ of spherical nano- $\mathrm{SiO}_{2}$ were added, and mass fraction of $0.5 \%$ of the coupling agent was added, and then they were fully mixed using a magnetic stirrer to prepare the coating agent, sealing preservation.

(3) The coating thickness of $0.5 \mathrm{~mm}$ was confirmed (coated with the small sample coating machine), before baking for 1 hour at $180^{\circ} \mathrm{C}$. The coated fabric 


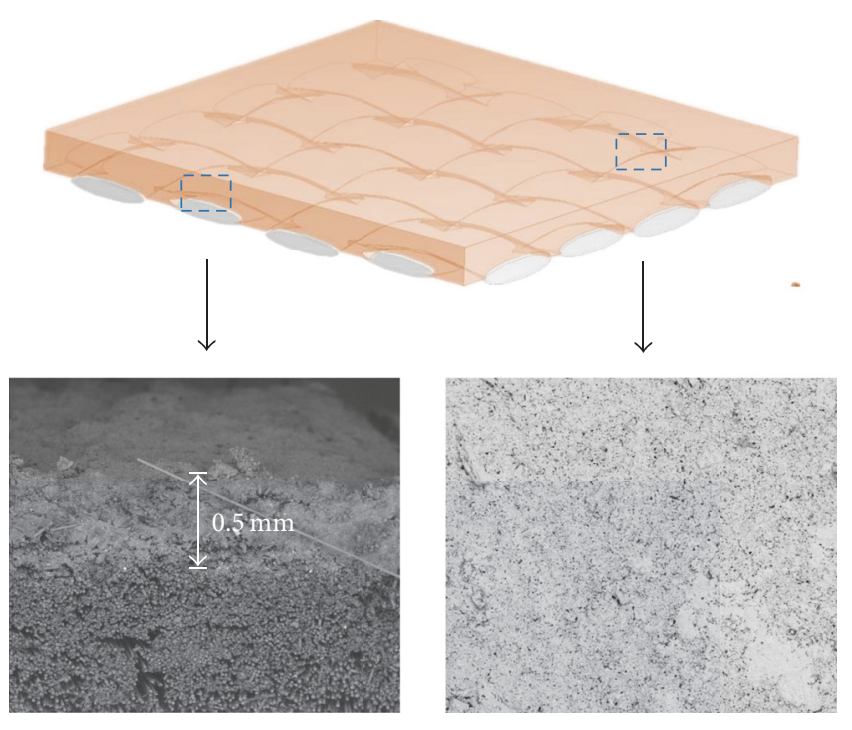

FIgURE 2: The micrograph of spherical nano- $\mathrm{SiO}_{2}$ coated fabrics.

sample was placed in the sample bag, after marking, and preparation of coated fabric was complete.

Figure 2 was the micrograph of prepared spherical nano$\mathrm{SiO}_{2}$ coated fabrics by the preparation process in Figure 1.

\subsection{Test Indexes and Methods}

(1) The Determination of Spherical Nano-SiO ${ }_{2}$ Coated Fabrics' Surface Microstructures. The determination of spherical nano- $\mathrm{SiO}_{2}$ coated fabrics' surface microstructures was performed using a field emission-scanning electron microscopy.

(2) The Performance Test for the Reflectivity of Thermal Radiation. The reflectivity of thermal radiation of the coated fabrics in the wavelength range $400-2000 \mathrm{~nm}$ was tested using a Lambda 750 UV-Vis near-infrared spectrophotometer, with step length $2 \mathrm{~nm}$.

(3) The Measurement of X-Ray Diffraction Spectra (XRD). The microscopic composition and crystalline state of coated fabrics were analyzed using a D8 Advance X-ray diffractometer (copper target, tube voltage $40 \mathrm{KV}$, tube current $40 \mathrm{~mA}$, and scanning range $10-80^{\circ}$ ) produced by BRUKER Company.

(4) Thermogravimetric Analysis (TG). The thermal stability properties of the coated fabrics were analyzed using a STA 409 PC thermogravimetric analyzer; the temperature range was $36-1200^{\circ} \mathrm{C}$, the heating rate was $10 \mathrm{~K} / \mathrm{min}$, and the test environment was atmospheric air.

(5) X-Ray Photoelectron Spectroscopy Analysis (XPS). The elemental compositions and chemical states of the relevant elements of the treated sample which had been heated at $1200^{\circ} \mathrm{C}$ were measured by $\mathrm{K}$-alpha X-ray photoelectron spectroscope.

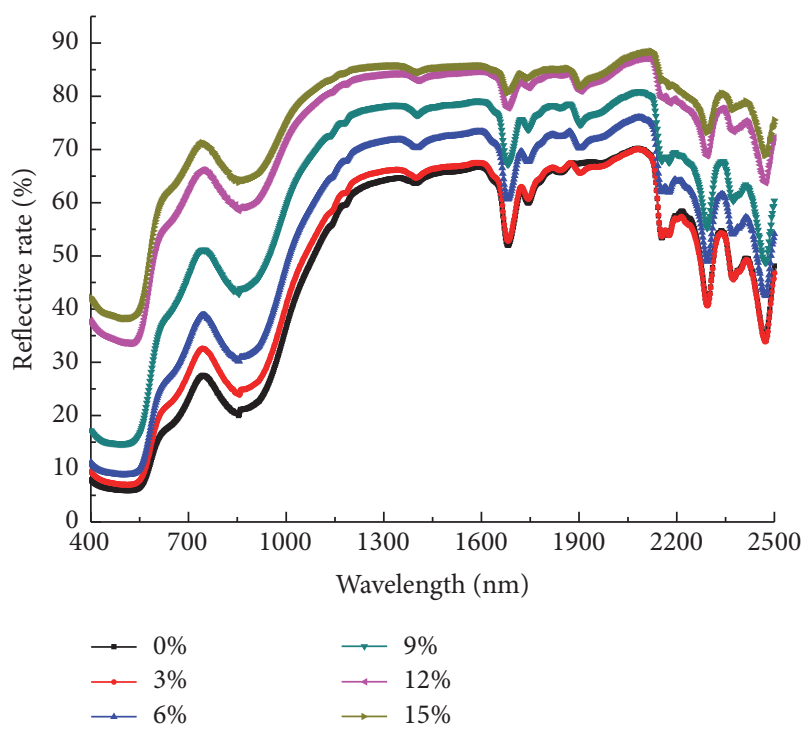

FIgURE 3: The UV-Vis spectra of different mass fraction of spherical nano- $\mathrm{SiO}_{2}$ coated fabrics.

(6) Energy Dispersive Spectrometer Analysis (EDS). The elemental distribution on the surface of the coated fabric at magnification of 60,000 times was analyzed qualitatively using an APOLLO XL ice characteristic X-ray spectrometer.

\section{Results and Discussion}

3.1. The Reflectivity of the Thermal Radiation of the Coated Fabrics. To explore the influence of the spherical nano- $\mathrm{SiO}_{2}$ mass fraction on the thermal radiation reflectivity of coated fabrics, the values of reflectivity for each sample in the wavelength range $400-2000 \mathrm{~nm}$ were tested using a Lambda 750 ultraviolet visible (UV-Vis) near-infrared spectrophotometer, and the test results are displayed in Figure 3.

As shown in Figure 3, in the wavelength range $400-2500 \mathrm{~nm}$, the reflectivity of coated fabrics increases as the mass fraction of spherical nano- $\mathrm{SiO}_{2}$ increases; when the mass fraction of spherical nano- $\mathrm{SiO}_{2}$ is $15 \%$, the reflectivity of coated fabrics is at its greatest. The data for the reflectivity of the thermal radiation with different mass fractions of spherical nano- $\mathrm{SiO}_{2}$ are summarized in Table 1 .

As shown in Table 1, as the mass percentage of spherical nano- $\mathrm{SiO}_{2}$ increases, the values of reflectivity for thermal radiation of the coated fabrics in the visible and near-infrared regions improve markedly. When the mass percentage of spherical nano- $\mathrm{SiO}_{2}$ is $15 \%$, the average value of reflectivity for the thermal radiation of coated fabrics was $74.30 \%$; within this, the average values of reflectivity in the visible and near-infrared bands were $52.16 \%$ and $80.75 \%$, respectively. Compared to the coated fabrics with no added packing $\left(0 \% \mathrm{SiO}_{2}\right)$, when the mass fraction of spherical nano- $\mathrm{SiO}_{2}$ was $15 \%$, the average values of reflectivity in the visible and near-infrared regions increased by $38.59 \%$ and $26.20 \%$, respectively. As a result, the average values of the reflectivity over the whole range increased by $28.98 \%$. 
TABLE 1: The reflectivity of the thermal radiation for different mass fractions of spherical nano- $\mathrm{SiO}_{2}$ coated fabrics.

\begin{tabular}{|c|c|c|c|}
\hline \multirow[b]{2}{*}{ Mass fraction } & \multicolumn{3}{|c|}{$\begin{array}{l}\text { Reflectivity of each sample in each } \\
\text { different wavelength range (\%) }\end{array}$} \\
\hline & $\begin{array}{l}\text { Visible region } \\
400-760 \mathrm{~nm}\end{array}$ & $\begin{array}{l}\text { Near-infrared } \\
\text { region } \\
760-2000 \mathrm{~nm}\end{array}$ & $\begin{array}{l}\text { Thermal } \\
\text { radiation } \\
400-2000 \mathrm{~nm}\end{array}$ \\
\hline $0 \%$ & 13.57 & 54.55 & 45.32 \\
\hline $3 \%$ & 16.45 & 56.31 & 47.33 \\
\hline $6 \%$ & 20.09 & 62.46 & 52.91 \\
\hline $9 \%$ & 29.09 & 70.15 & 60.89 \\
\hline $12 \%$ & 47.00 & 78.55 & 71.44 \\
\hline $15 \%$ & 52.16 & 80.75 & 74.30 \\
\hline
\end{tabular}

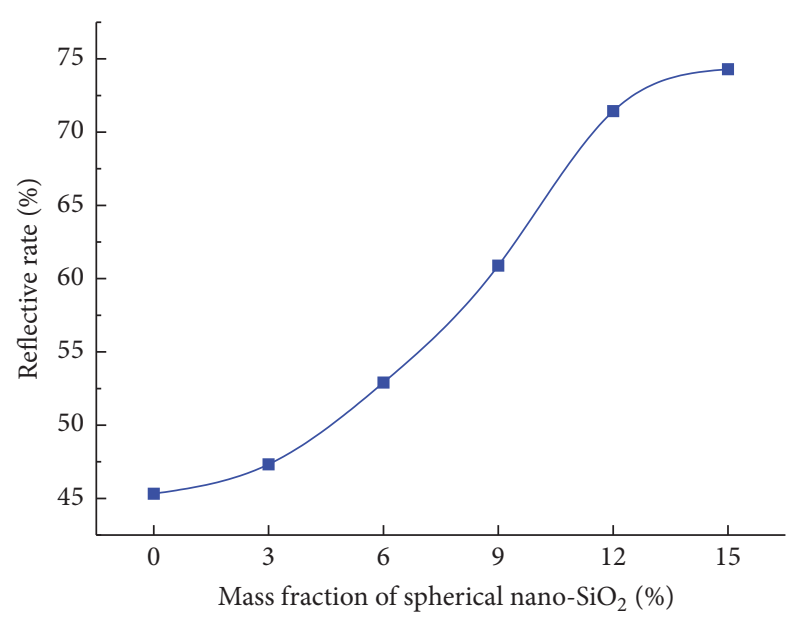

FIGURE 4: Change of reflectivity for the thermal radiation of coated fabrics against mass percentage of spherical nano- $\mathrm{SiO}_{2}$.

Figure 4 shows the change trend for the reflectivity for the thermal radiation of coated fabrics as the mass percentage of spherical nano- $\mathrm{SiO}_{2}$ is increased. It shows that, with increasing spherical nano- $\mathrm{SiO}_{2}$ content, the average values of reflectivity initially improve significantly, and after the mass percentage of spherical nano- $\mathrm{SiO}_{2}$ reaches $12 \%$, the growth rate of the reflectivity gradually slows and the curve tends to flatten.

The main cause of the phenomena is the regular changes in coated fabrics' surfaces with the increase of $\mathrm{SiO}_{2}$ content. As shown in Figures 5(a)-5(f), there are a certain growing number of nanoscale bulbiform bulges in coated fabrics' surfaces. Since the surface flatness of the coated fabrics drops gradually and the number of surface deformation increases, the contact surfaces between incoming lights and coated fabrics' surfaces expand, and thermal radiation reflectivity effect improves.

According to the theory of photon scattering, after incident light reaches the surface of the material, the energy is divided by three different mechanisms simultaneously: some photons hitting the surface of materials undergo reflection, refraction, and other scattering processes and return to the space of the incident radiation; other photons are absorbed by the material, and the energy is converted into molecular vibrational and rotational energy; the remaining photons go through the material, by transmission, and continue to transmit into the next medium [20-22]. The thickness of the prepared coated fabrics was about $2 \mathrm{~mm}$ and the transmission rate of photons was almost zero. Therefore, the smaller the absorption rate of photons by the coated fabrics in the range 400-2000 nm, the higher the reflectivity of the thermal radiation. The absorption mechanism for the photons is mainly decided by the width of the forbidden band of the material itself; as long as the photon energy is larger than the width of the forbidden band of the material, then the material can undergo the transition between the top of the valence band and the bottom of the conduction band [23-25].

For the absorption of photons by this band, the energy of the photons representing the region of the width of the forbidden band can be calculated using

$$
E_{g}=h v=\frac{h c}{\lambda}
$$

where $E_{g}$ is the energy gap, $v$ is the frequency occurring in the transition, and $\lambda$ is the wavelength of the corresponding photons [26-28].

According to the calculation, the width range of the forbidden band corresponding to the absorption wavelength range $400-2000 \mathrm{~nm}$ is $0.62-3.1 \mathrm{eV}[29,30]$; the width of the forbidden band of particles themselves for materials located in this range will show the absorption for the thermal radiation. The width of the forbidden band for spherical nano- $\mathrm{SiO}_{2}$ adopted by the experimental group is up to $8.8 \mathrm{eV}$, indicating it is optically inert and has no absorption effect on the thermal radiation [31,32]. Therefore, the reflectivity of thermal radiation for prepared coated fabrics increases significantly with increasing mass fraction of spherical nano$\mathrm{SiO}_{2}$.

At the same time, because the nano- $\mathrm{SiO}_{2}$ used in the experiment is spherical, based on the theories of tightness and stacking of KM (Kubelka and Munk), the maximum stacking density and apparent density (apparent density of powders) of standard spherical objects can be achieved [12]. When using similar particle sizes, the surface area is largest when packing spherical particles, and the reflection intensity of particles of light is the strongest. In the coating system used here, the gaps between particles of spherical nano- $\mathrm{SiO}_{2}$ are very small and the degree of chaos of stacking is small. The viscosity of powders is strong and the liquidity of powders is stronger when preparing the coating, which results in a coating with excellent properties. Therefore, when compared with other morphologies of $\mathrm{SiO}_{2}$ packing, spherical nano- $\mathrm{SiO}_{2}$ results in functional packing which reflects the thermal radiation most efficiently [33].

In addition, in the visible light region of $400-760 \mathrm{~nm}$, the reflectivity of thermal radiation by coated fabrics is significantly improved. This may be due to the increase of the mass percentage of spherical nano- $\mathrm{SiO}_{2}$ with an average particle size of $20 \mathrm{~nm}$, which gradually fill the pores in the coating system. Particles in the coating system with each function produce a synergistic effect, and the ability of coated 


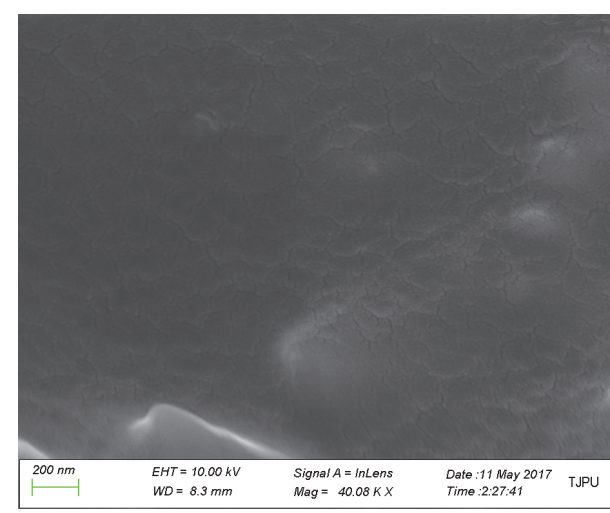

(a)

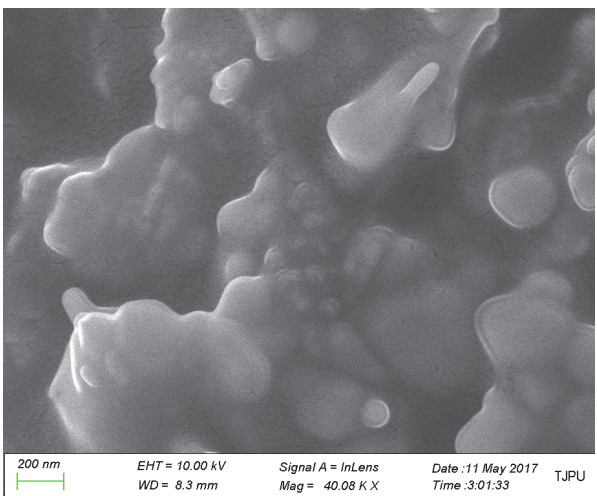

(c)

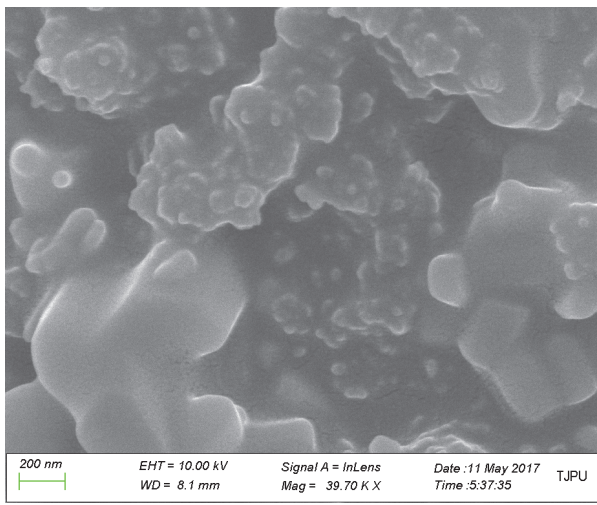

(e)

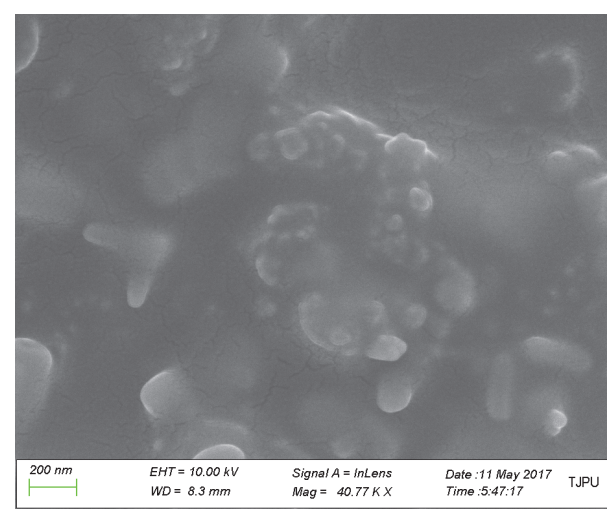

(b)

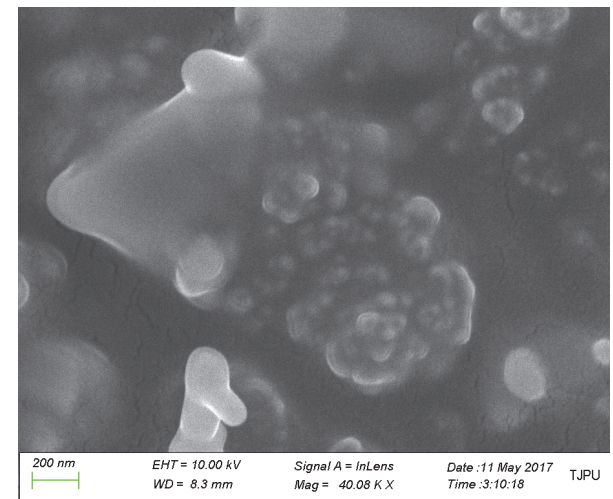

(d)

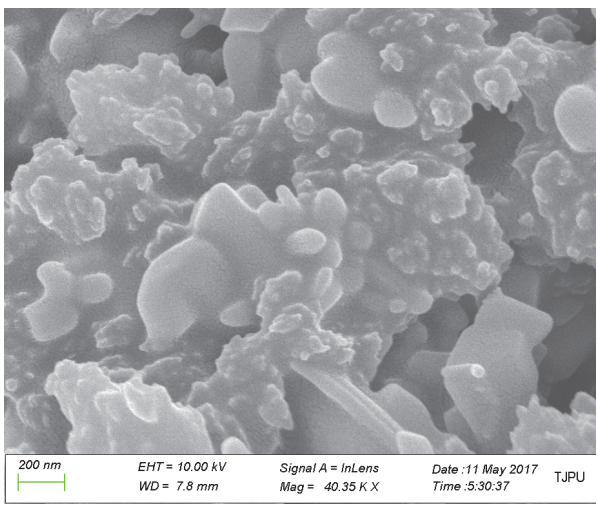

(f)

FIGURE 5: SEM photographs of spherical nano- $\mathrm{SiO}_{2}$ coated fabrics' surface microstructures. (a) Mass percentage of spherical nano-SiO ${ }_{2}$ is $0 \%$; (b) mass percentage of spherical nano- $\mathrm{SiO}_{2}$ is $3 \%$; (c) mass percentage of spherical nano- $\mathrm{SiO}_{2}$ is $6 \%$; (d) mass percentage of spherical nano- $\mathrm{SiO}_{2}$ is $9 \%$; (e) mass percentage of spherical nano- $\mathrm{SiO}_{2}$ is $12 \%$; (f) mass percentage of spherical nano- $\mathrm{SiO}_{2}$ is $15 \%$.

fabrics to reflect thermal radiation is significantly increased [34].

To explore the influence of spherical nano- $\mathrm{SiO}_{2}$, and other functional packing, on the reflectivity of thermal radiation by coated fabrics, further work needs to be carried out on the microstructure and the structure of particles and their influence on the packing of coated fabrics.

3.2. Analysis of the XRD Test Results for the Coated Fabrics. By analysis of the X-ray diffraction patterns, the internal micrograin varieties of spherical nano- $\mathrm{SiO}_{2}$ coated fabrics were identified and their crystalline states were further analyzed. Also, a microlevel explanation of the ability of coated fabrics to reflect thermal radiation was proposed.

The X-ray diffraction patterns for spherical nano- $\mathrm{SiO}_{2}$ coated fabrics were measured using a D8 Advance X-ray diffractometer, and results are presented in Figure 6.

Figure 6 shows the X-ray diffraction patterns for spherical nano- $\mathrm{SiO}_{2}$ coated fabrics, comparing the sample atlas with the standard atlas of $\mathrm{SiO}_{2}$. There is a strong diffraction 


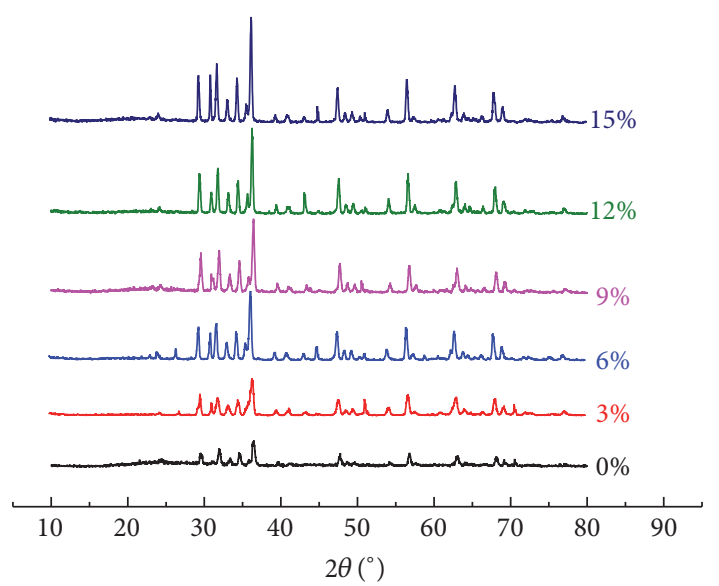

FIGURE 6: X-ray diffraction patterns for spherical nano- $\mathrm{SiO}_{2}$ coated fabrics.

peak at $2 \theta=34.44^{\circ}$, and the peak shape is clear, sharp, and symmetrical. Through analysis of the diffraction pattern using the MDI Jade 6 software, the interplanar spacing of spherical nano- $\mathrm{SiO}_{2}$ in the samples is found to be $2.60 \mathrm{~nm}$, which is equal to the value of the response interplanar spacing of $2.60 \mathrm{~nm}$ in the standard atlas of $\mathrm{SiO}_{2}$. Therefore, according to results for the characteristic peak and the data for the interplanar spacing, the $\mathrm{SiO}_{2}$ crystal structure contained in the coated fabrics is thought to be the same as $\mathrm{SiO}_{2}(\mathrm{PDF} \# 46-$ 1242).

As shown in Figure 7, the strengths of the $\mathrm{SiO}_{2}$ diffraction peaks of the coated fabrics increase with the rises of mass percentage of spherical nano- $\mathrm{SiO}_{2}$ at $34.44^{\circ}$. This demonstrates the content increase of nano- $\mathrm{SiO}_{2}$ on the surface of coated fabrics.

When thermal radiation is incident on the crystals, the reflection and the refraction occur on the crystal boundary, and the larger the grain size and the closer the grains, the greater the extent of reflection and refraction and the stronger the ability to reflect the thermal radiation [35]. A fitting analysis of the X-ray diffraction pattern of spherical nano$\mathrm{SiO}_{2}$ coating using MDI Jade 6 software was carried out, and the average grain sizes in the samples of spherical nano- $\mathrm{SiO}_{2}$ coated fabrics are summarized in Figure 8.

Figure 8 shows that, with the increasing of spherical nano- $\mathrm{SiO}_{2}$ content, the average grain sizes are on the rise. When the mass percentage of spherical nano- $\mathrm{SiO}_{2}$ is $15 \%$, the average grain size in the samples of spherical nano$\mathrm{SiO}_{2}$ coated fabrics was $33 \mathrm{~nm}$, which is $15.6 \mathrm{~nm}$ larger than when no spherical nano- $\mathrm{SiO}_{2}$ is added. So, after adding spherical nano- $\mathrm{SiO}_{2}$, the average grain size increased by $89.66 \%$, and the ability to reflect thermal radiation increased by $63.95 \%$. This could be because, after adding spherical nano- $\mathrm{SiO}_{2}$, a synergistic effect occurs among particles in the coating, influenced by the preparation method and other external factors, and the crystal bridge at points of mutual contact among grains, and the grain size increases; as the combination of the grains becomes closer, the ability to reflect the thermal radiation improves significantly [36].

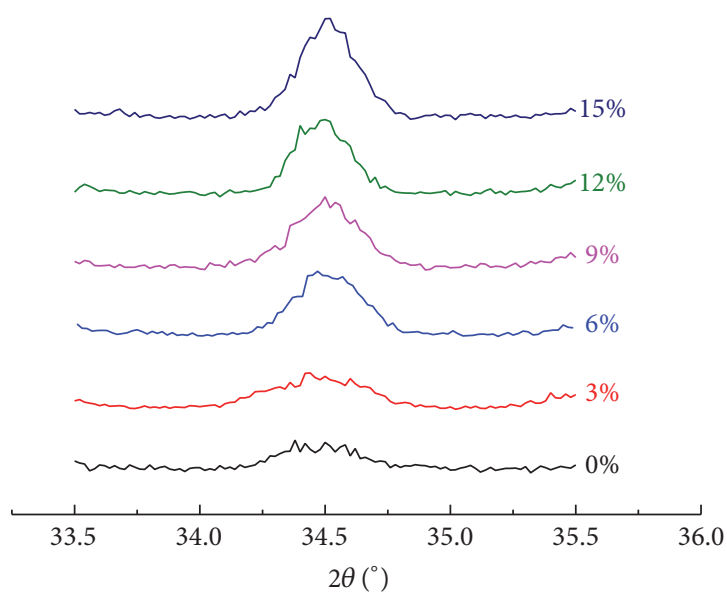

FIGURE 7: Change of the $\mathrm{SiO}_{2}$ diffraction intensities against mass percentage of spherical nano- $\mathrm{SiO}_{2}$.

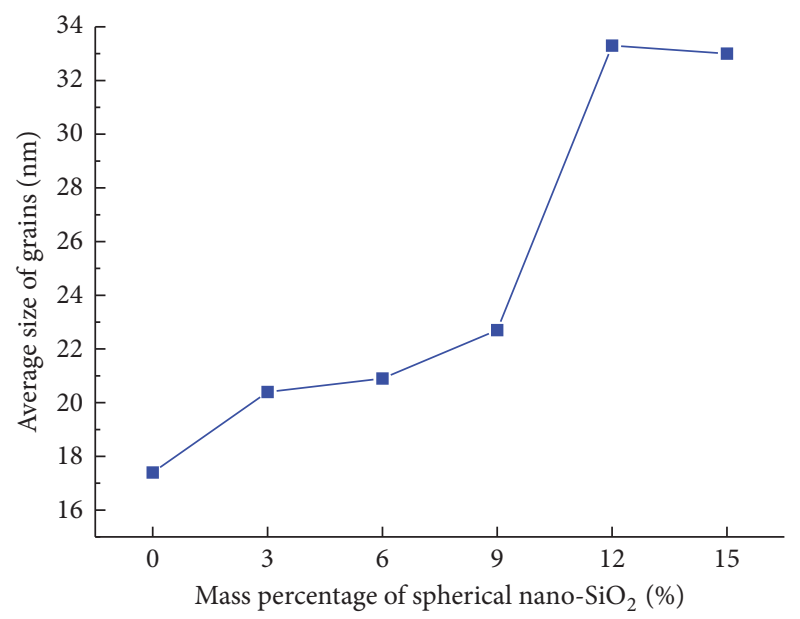

FIGURE 8: Change of the average grain size of coated fabrics against mass percentage of spherical nano- $\mathrm{SiO}_{2}$.

3.3. Analysis of the TG Test Results of the Coated Fabrics. Thermal stability at high temperature is one of the most important indicators to assess the ablation resistance of coated fabrics. To test the thermal stability of prepared spherical nano- $\mathrm{SiO}_{2}$ coated fabrics at high temperature, the thermogravimetric (TG) and differential thermal analysis (DTA) of the prepared coated fabrics were carried out using an STA 409 PC thermogravimetric analyzer. The temperature range tested was $36-1200^{\circ} \mathrm{C}$, the heating rate was $10 \mathrm{~K} / \mathrm{min}$, and the test atmosphere was air. Figure 9 shows the TG-DTA curve test results for spherical nano- $\mathrm{SiO}_{2}$ coated fabrics.

From Figure 9, it can be seen that, in the process of the temperature increasing to $1200^{\circ} \mathrm{C}$, there is no apparent phase where the weight is constant. The weight reduces gradually until $1200^{\circ} \mathrm{C}$, and the residual mass is $88.49 \%$, displaying excellent thermal stability. At $112.50^{\circ} \mathrm{C}$, the weight loss of the coating is $0.92 \%$, most likely due to evaporation of moisture in the coating matrix and the decomposition of a small amount of solvent, silicon hydroxyl, and small molecule polymers in the organic silicon resin, corresponding to the 


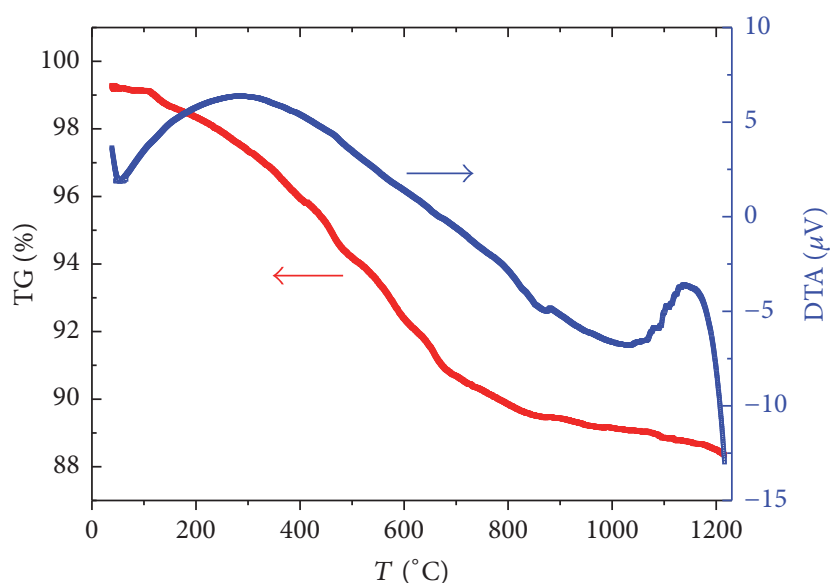

FIgURE 9: TG-DTA curves for spherical nano- $\mathrm{SiO}_{2}$ coated fabrics.

endothermic peak in the DTA curve at $53.22^{\circ} \mathrm{C}$. At $285.37^{\circ} \mathrm{C}$ there is a relatively wide exothermic peak in the DTA curve, due to the oxidation reaction of the coating matrix of silicone resin with oxygen in the testing atmosphere. The thermal decomposition of coated fabrics is the most intense when the temperature is gradually increased to $463.79^{\circ} \mathrm{C}$; at this temperature the predominant process is the thermal decomposition of C-C bonds, $\mathrm{C}-\mathrm{H}$ bonds, benzene pyrolysis, and other organic groups on the silicone resin as the coating matrix [37]. At this temperature, the weight loss of the coated fabric is $5.14 \%$ and when the temperature is $1138.72^{\circ} \mathrm{C}$ an unapparent exothermic peak occurred in the DTA curve. This is due to the exothermic reaction of spherical nano$\mathrm{SiO}_{2}$ with the $\mathrm{CaO}$ which occurs at high temperature and generates $\mathrm{CaSiO}_{3}$. The $\mathrm{CaO}$ is produced by the exothermic decomposition of $\mathrm{CaCO}_{3}$ filler around $900^{\circ} \mathrm{C}$. The highresolution XPS spectra of the treated sample heated at $1200^{\circ} \mathrm{C}$ are shown in Figure 10. From the high-resolution spectra of $\mathrm{Ca} 2 \mathrm{p}$, it is observed that $\mathrm{Ca}$ is mainly present in the form of $\mathrm{CaSiO}_{3}$, with binding energy of approximately $347.05 \mathrm{eV}$, indicating a Ca-O bond of $\mathrm{CaSiO}_{3}$ [38]. In addition, two small peaks with binding energy of $351.2 \mathrm{eV}$ and $350.3 \mathrm{eV}$ are detected for the coated fabric, which is ascribed to a small amount of $\mathrm{CaO}$. Figure 10(c) shows the Si 2p XPS spectra. It could be divided into two components with binding energies of $102.1 \mathrm{eV}$ and $103.3 \mathrm{eV}$, which are, respectively, ascribed to $\mathrm{CaSiO}_{3}$ and a small amount of $\mathrm{SiO}_{2}$ [39]. The XPS survey spectra analysis results prove the existence of $\mathrm{CaSiO}_{3}$ in the treated sample which has been heated at $1200^{\circ} \mathrm{C}$.

Figures 11(a)-11(f) show the contrast between the TG curves of coated fabrics before and after adding spherical nano- $\mathrm{SiO}_{2}$ with different mass fractions. It can be seen from Figures $11(\mathrm{a})-11(\mathrm{f})$ that, after adding the functional packing, the weight loss of the coated fabrics is decreased gradually with the mass fraction growth of the spherical nano- $\mathrm{SiO}_{2}$. When the temperature is increased to $1200^{\circ} \mathrm{C}$, the coated fabric without added packing is found to have lost $30.26 \%$ of its weight, whereas the coated fabric with added $15 \%$ of the packing loses $11.49 \%$. The difference in these values, of $18.77 \%$, is because, after adding the spherical nano- $\mathrm{SiO}_{2}$, the

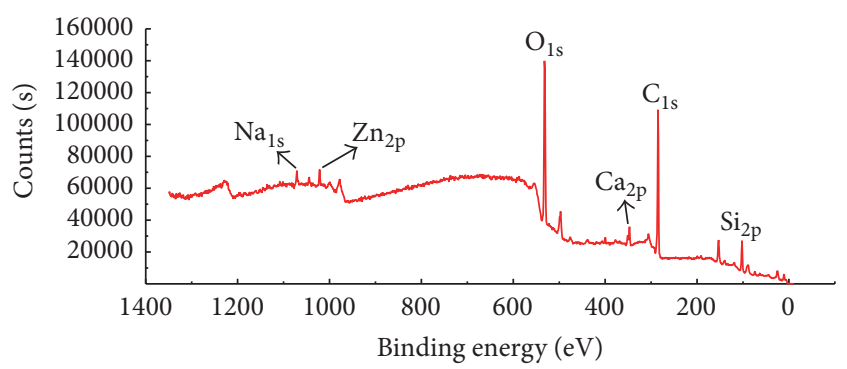

(a)

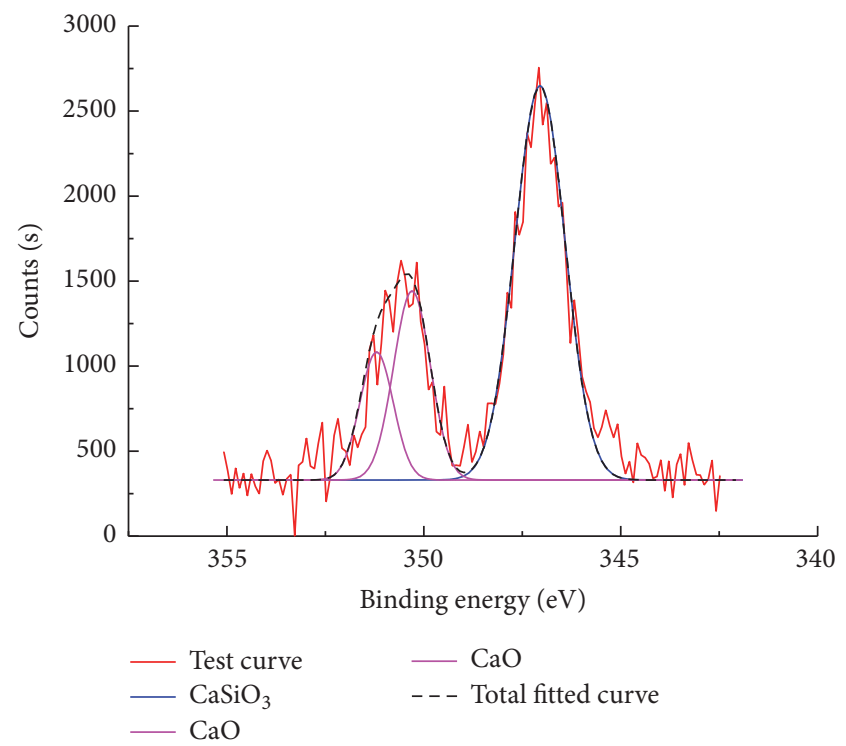

(b)

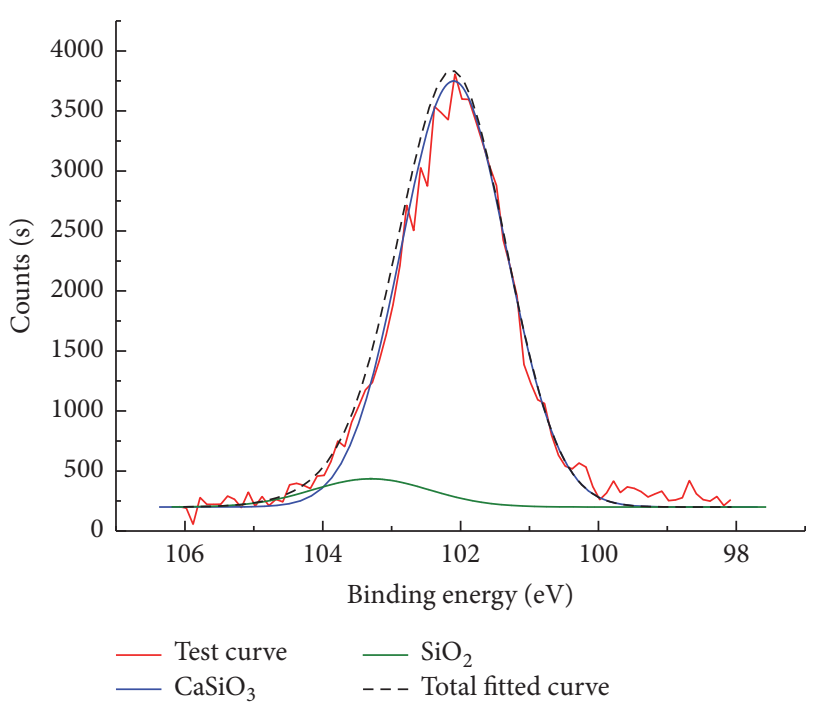

(c)

FIGURE 10: XPS survey spectra of treated sample. (a) XPS survey spectra; (b) Ca 2p XPS spectra; (c) Si 2p XPS spectra.

thermal stability of the coated fabric is greatly improved. This may be because spherical nano- $\mathrm{SiO}_{2}$ has high surface activity, which has an affinity for moisture in the air, forming hydroxyl groups. Also, there may be physical or chemical crosslinking 


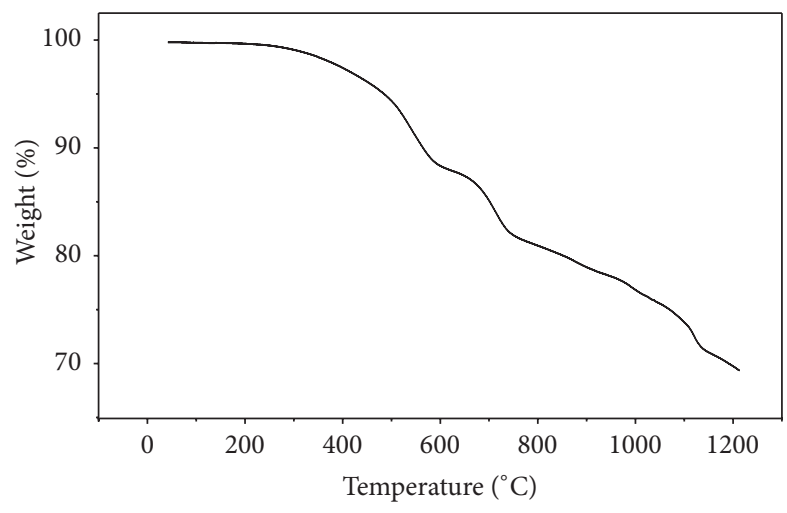

(a)

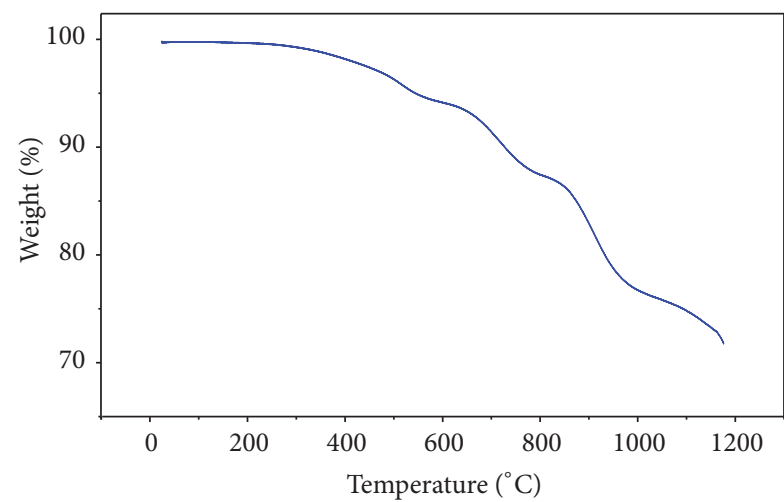

(c)

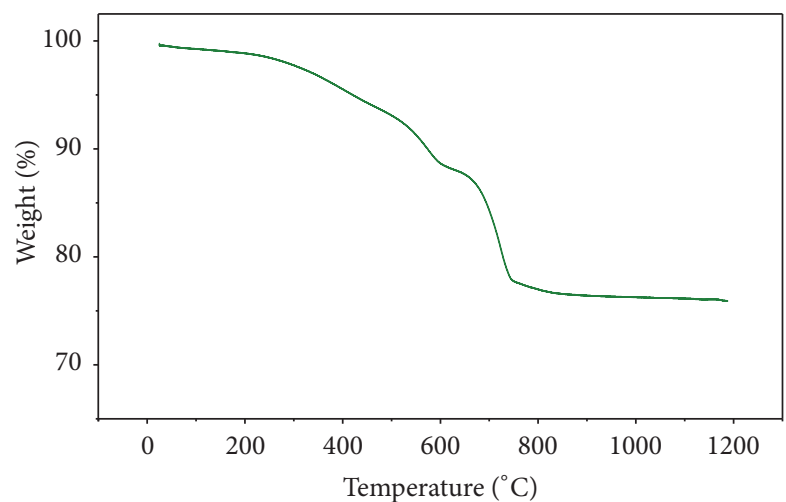

(e)

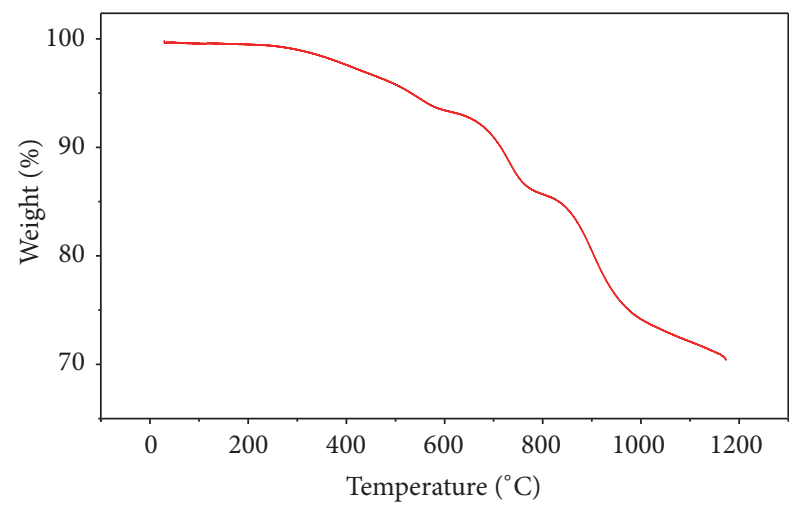

(b)

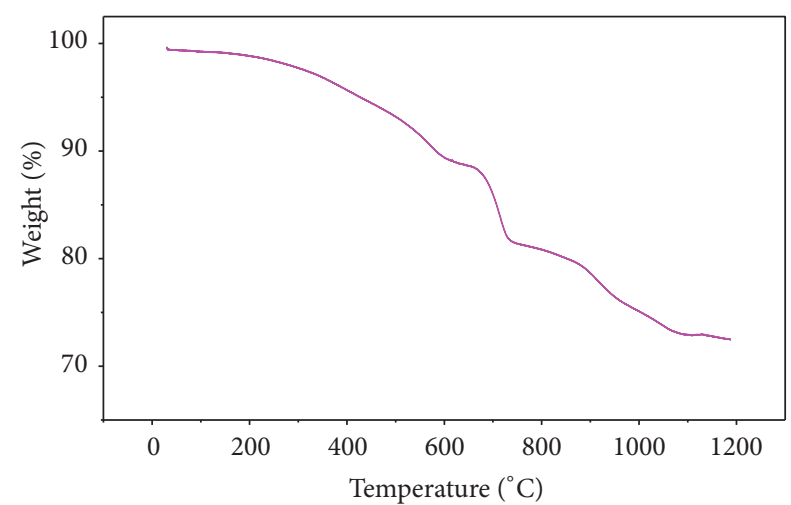

(d)

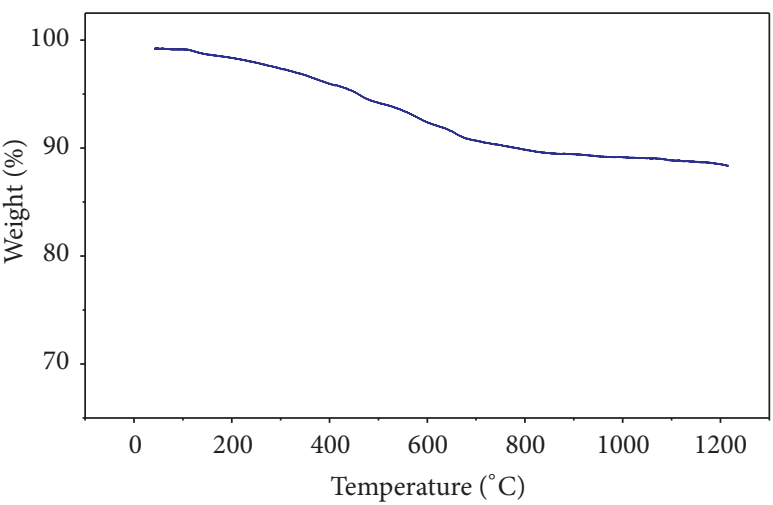

(f)

FIGURE 11: TG curves for spherical nano- $\mathrm{SiO}_{2}$ coated fabrics. (a) Mass percentage of spherical nano-SiO 2 is $0 \%$; (b) mass percentage of spherical nano- $\mathrm{SiO}_{2}$ is $3 \%$; (c) mass percentage of spherical nano- $\mathrm{SiO}_{2}$ is $6 \%$; (d) mass percentage of spherical nano- $\mathrm{SiO}_{2}$ is $9 \%$; (e) $\mathrm{mass}$ percentage of spherical nano- $\mathrm{SiO}_{2}$ is $12 \%$; (f) mass percentage of spherical nano- $\mathrm{SiO}_{2}$ is $15 \%$.

such as hydrogen bonds between spherical nano- $\mathrm{SiO}_{2}$ and the coating matrix or other functional packing, which serves to enhance the binding force of each functional particle in the coating system, and it slows down the decomposition of other active monomers, small molecules, and other constituent parts in the coatings system [40]. At the same time, because spherical nano- $\mathrm{SiO}_{2}$ has excellent high temperature resistance, it serves to shield oxygen and heat in the external environment and hinders the transfer of volatile degradation products on the surface of coated fabrics. Due to the reasons outlined, adding spherical nano- $\mathrm{SiO}_{2}$ has a positive effect in significantly improving the thermal stability of coated fabrics at high temperature [41].

3.4. EDS Analysis of the Surface of Coated Fabrics. To explore the influence of elemental changes of coated fabrics on the reflectivity of thermal radiation before and after adding spherical nano- $\mathrm{SiO}_{2}$, an X-ray spectrometer with the characteristic of cooling the fields was used. The coated fabrics were imaged at magnification of 60,000 times, and the distribution 


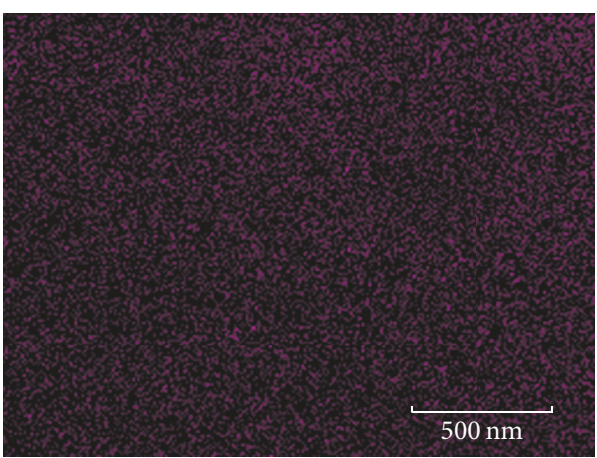

(a) Before adding nano- $\mathrm{SiO}_{2}$

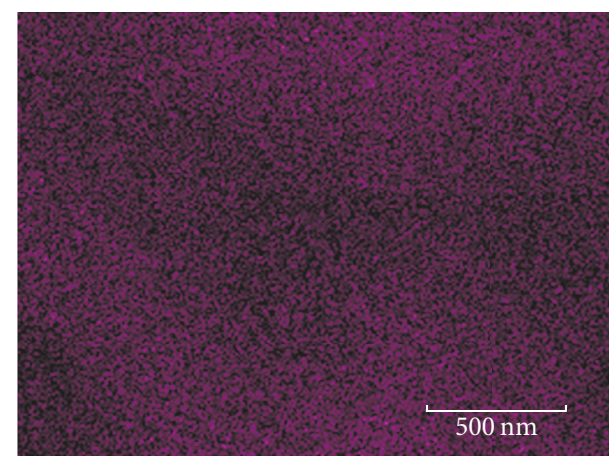

(b) After adding nano-SiO

FIGURE 12: Distribution of $\mathrm{Si}$ in the coated fabrics before and after adding spherical nano- $\mathrm{SiO}_{2}$ with mass fraction of $15 \%$.

of elements in the same areas was established. Figure 12 shows the distribution of $\mathrm{Si}$ in the coated fabric samples before and after adding spherical nano- $\mathrm{SiO}_{2}$ with mass fraction of $15 \%$.

Figure 12 shows that, before adding spherical nano$\mathrm{SiO}_{2}$ to the coated fabric, the $\mathrm{Si}$ element is relatively evenly distributed in the coating system; the main source of the $\mathrm{Si}$ is from talcum powder used as the packing. After adding spherical nano- $\mathrm{SiO}_{2}$, the distribution intensity of $\mathrm{Si}$ is increased significantly; the $\mathrm{Si}$ source is now from the two types of packing-talcum powder and spherical nano- $\mathrm{SiO}_{2}$. However, before and after adding spherical nano- $\mathrm{SiO}_{2}$, the contents of $\mathrm{Ca}, \mathrm{Fe}, \mathrm{Mg}$, and $\mathrm{Zn}$ in the samples are not changing. Therefore, the EDS qualitative analysis shows that, after adding spherical nano- $\mathrm{SiO}_{2}$, the content of $\mathrm{Si}$ in the system is increased significantly. This helps to explain that the increase in ability to reflect the thermal radiation and the increased Si content in the system are closely linked. In essence, the increase in reflectivity of thermal radiation for coated fabrics is mainly due to the addition of spherical nano- $\mathrm{SiO}_{2}$.

\section{Conclusions}

(1) In the wavelength range $400-2000 \mathrm{~nm}$, the reflectivity of thermal radiation for coated fabrics is evidently increased as the mass fraction of spherical nano$\mathrm{SiO}_{2}$ is increased; when the mass fraction of added spherical nano- $\mathrm{SiO}_{2}$ is $15 \%$, the reflectivity of coated fabrics is the greatest, with an average value of $74.30 \%$.

(2) The average size of grains in prepared coated fabrics was $33 \mathrm{~nm}$, which was $15.6 \mathrm{~nm}$ larger than those with no added spherical nano- $\mathrm{SiO}_{2}$. After adding the spherical nano- $\mathrm{SiO}_{2}$, the average grain size in the samples increased by $89.66 \%$, and the ability to reflect thermal radiation increased by $63.95 \%$.

(3) When heating the coating system to $1200^{\circ} \mathrm{C}$, there was no apparent weight loss stage. The residual mass in the coating system was as high as $88.49 \%$ after adding $15 \%$ spherical nano- $\mathrm{SiO}_{2}$, exhibiting excellent thermal stability.

\section{Conflicts of Interest}

The authors declare that there are no conflicts of interest regarding the publication of this paper.

\section{References}

[1] H. Barani, "Preparation of antibacterial coating based on in situ synthesis of $\mathrm{ZnO} / \mathrm{SiO}_{2}$ hybrid nanocomposite on cotton fabric," Applied Surface Science, vol. 320, pp. 429-434, 2014.

[2] M. Liu, T. Xue, Y. Zhao, S. Liu, and J. Liu, "Composite thermal protection coating on woven silica fiber fabrics," Science China Technological Sciences, vol. 57, no. 6, pp. 1121-1126, 2014.

[3] Z. Fanglong, X. Qun, F. Qianqian, L. Rangtong, and L. Kejing, "Influence of nano-silica on flame resistance behavior of intumescent flame retardant cellulosic textiles: remarkable synergistic effect?" Surface and Coatings Technology, vol. 294, pp. 90-94, 2016.

[4] S. S. Palaskar, A. N. Desai, and S. R. Shukla, "Development of multifunctional cotton fabric using atmospheric pressure plasma and nano-finishing," Journal of the Textile Institute, vol. 107, no. 3, pp. 405-412, 2016.

[5] W. Owens, D. Merkel, F. Sansoz, and D. Fletcher, "Fracture behavior of woven silicon carbide fibers exposed to hightemperature nitrogen and oxygen plasmas," Journal of the American Ceramic Society, vol. 98, no. 12, pp. 4003-4009, 2015.

[6] F. Branda, G. Malucelli, M. Durante et al., "Silica treatments: a fire retardant strategy for hemp fabric/epoxy composites," Polymers, vol. 8, no. 8, article 313, 2016.

[7] P. Zhao, S. Liu, K. Xiong, W. Wang, and Y. Liu, "Highly flame retardancy of cotton fabrics with a novel phosphorus/nitrogen/silicon flame-retardant treating system," Fibers and Polymers, vol. 17, no. 4, pp. 569-575, 2016.

[8] T. P. Coons, J. W. Reutenauer, A. Mercado, M. A. Kmetz, and S. L. Suib, "The characterization of an oxide interfacial coating for ceramic matrix composites," Materials Science and Engineering A, vol. 573, pp. 190-196, 2013.

[9] Y. Takizawa and D. D. L. Chung, "Through-thickness thermal conduction in glass fiber polymer-matrix composites and its enhancement by composite modification," Journal of Materials Science, vol. 51, no. 7, pp. 3463-3480, 2016. 
[10] Q. Zhang, W. Zhang, J. Huang et al., "Flame retardance and thermal stability of wool fabric treated by boron containing silica sols," Materials and Design, vol. 85, pp. 796-799, 2015.

[11] X. Zhu, Y. Wu, C. Tian, Y. Qing, and C. Yao, "Synergistic effect of nanosilica aerogel with phosphorus flame retardants on improving flame retardancy and leaching resistance of wood," Journal of Nanomaterials, vol. 2014, Article ID 867106, 9 pages, 2014.

[12] Y. Wang, X. Gao, P. Chen et al., "Preparation and thermal performance of paraffin/ $\mathrm{Nano}-\mathrm{SiO}_{2}$ nanocomposite for passive thermal protection of electronic devices," Applied Thermal Engineering, vol. 96, pp. 699-707, 2016.

[13] T. A. Nguyen, H. Nguyen, T. V. Nguyen, H. Thai, and X. Shi, "Effect of nanoparticles on the thermal and mechanical properties of epoxy coatings," Journal of Nanoscience and Nanotechnology, vol. 16, no. 9, pp. 9874-9881, 2016.

[14] Y. Li, T. Zhao, X. Qu, H. Ding, and F. Li, "Synthesis of waterborne polyurethane modified by nano- $\mathrm{SiO}_{2}$ silicone and properties of the WPU coated RDX," China Petroleum Processing and Petrochemical Technology, vol. 17, no. 2, pp. 39-45, 2015.

[15] X.-Y. Yang, Y. Yue, J.-H. Cai, Y. Liu, and X.-M. Wu, "Experimental study and stabilization mechanisms of silica nanoparticles based brine mud with high temperature resistance for horizontal shale gas wells," Journal of Nanomaterials, vol. 2015, Article ID 745312, 9 pages, 2015.

[16] J.-W. Lee, K. Kim, S. B. Khan et al., "Synthesis, characterization, and thermal and proton conductivity evaluation of 2,5polybenzimidazole composite membranes," Journal of Nanomaterials, vol. 2014, Article ID 460232, 2014.

[17] C. Loka, K.-R. Park, and K.-S. Lee, " $\mathrm{SiO}_{2} / \mathrm{TiO}_{2} / \mathrm{n}-\mathrm{Si} / \mathrm{Ag}(\mathrm{Cr}) /$ $\mathrm{TiO}_{2}$ thin films with superhydrophilicity and low-emissivity," Japanese Journal of Applied Physics, vol. 55, Article ID 01AA06, 5 pages, 2016.

[18] A. Krimmer, E. Rosenthal, E. Andreenko et al., "Testing of a $\mathrm{SiO}_{2} / \mathrm{TiO}_{2}$ mirror coating on a stainless steel substrate under ITER in-port conditions," Fusion Engineering and Design, vol. 96-97, pp. 817-820, 2015.

[19] T. Fu, J. Tang, K. Chen, and F. Zhang, "Visible, near-infrared and infrared optical properties of silica aerogels," Infrared Physics and Technology, vol. 71, pp. 121-126, 2015.

[20] M.-M. Zhang, X.-L. Liu, T. Ma, and J.-F. Chen, "Preparation of silica aerogels and application in thermal insulation coating of fabric," Rare Metal Materials and Engineering, vol. 44, no. suppl1, pp. 421-425, 2015.

[21] A. Tavassolimanesh, A. Niknejad, and H. Assaee, "Effects of polyethylene and polyurethane Teflon-fillers on the energy absorption behavior of composite tubes during the flattening tests," Journal of Composite Materials, vol. 49, no. 17, pp. 20572072, 2015.

[22] M. Kara, M. Uyaner, and A. Avci, "Repairing impact damaged fiber reinforced composite pipes by external wrapping with composite patches," Composite Structures, vol. 123, pp. 1-8, 2015.

[23] A. H. Mohammed Ali and W. Yu, "Thermal protective performance of multilayer fire fighting fabric," International Journal of Clothing Science and Technology, vol. 26, no. 3, pp. 235-246, 2014.

[24] A. P. Mouritz, S. Feih, E. Kandare, and A. G. Gibson, "Thermalmechanical modelling of laminates with fire protection coating," Composites Part B: Engineering, vol. 48, pp. 68-78, 2013.

[25] Y. H. Yu, B. G. Kim, and D. G. Lee, "Cryogenic reliability of composite insulation panels for liquefied natural gas (LNG) ships," Composite Structures, vol. 94, no. 2, pp. 462-468, 2012.
[26] H. Liao, Y. Liu, J. Jiang, Y. Liu, and Q. Wang, "Flameretardant, glass-fabric-reinforced epoxy resin laminates fabricated through a gradient distribution mode," Journal of Applied Polymer Science, vol. 134, no. 2, 2017.

[27] X. Ding, F. Fang, T. Du et al., "Carbon nanotube-filled intumescent multilayer nanocoating on cotton fabric for enhancing flame retardant property," Surface and Coatings Technology, vol. 305, pp. 184-191, 2016.

[28] F. Fang, B. Tong, T. Du et al., "Unique nanobrick wall nanocoating for flame-retardant cotton fabric via layer-by-layer assembly technique," Cellulose, vol. 23, no. 5, pp. 3341-3354, 2016.

[29] R. K. Upadhyay, A. Dubey, P. R. Waghmare, R. Priyadarshini, and S. S. Roy, "Multifunctional reduced graphene oxide coated cloths for oil/water separation and antibacterial application," RSC Advances, vol. 6, no. 67, pp. 62760-62767, 2016.

[30] Z. Nooralian, M. Parvinzadeh Gashti, and I. Ebrahimi, "Fabrication of a multifunctional graphene/polyvinylphosphonic acid/cotton nanocomposite via facile spray layer-by-layer assembly," RSC Advances, vol. 6, no. 28, pp. 23288-23299, 2016.

[31] F. Carosio, G. Fontaine, J. Alongi, and S. Bourbigot, "Starchbased layer by layer assembly: efficient and sustainable approach to cotton fire protection," ACS Applied Materials and Interfaces, vol. 7, no. 22, pp. 12158-12167, 2015.

[32] R. Stopforth and S. Adali, "Thermal endurance testing of Kevlar fabric/phenolic sandwich composite protecting the electronics of a search and rescue robot," Advanced Composites Letters, vol. 23, no. 5, pp. 111-114, 2014.

[33] V. Goudarzi, I. Shahabi-Ghahfarrokhi, and A. Babaei-Ghazvini, "Preparation of ecofriendly UV-protective food packaging material by starch/ $\mathrm{TiO}_{2}$ bio-nanocomposite: characterization," International Journal of Biological Macromolecules, vol. 95, pp. 306-313, 2017.

[34] X. Y. Wang, J. X. Ma, C. G. Li, and H. X. Wang, "Thermosetting polyimide resin matrix composites with interpenetrating polymer networks for precision foil resistor chips based on special mechanical performance requirements," Applied Surface Science, vol. 299, pp. 73-80, 2014.

[35] F. Song, Q. Wang, and T. Wang, "High mechanical and tribological performance of polyimide nanocomposites reinforced by chopped carbon fibers in adverse operating conditions," Composites Science and Technology, vol. 134, pp. 251-257, 2016.

[36] W. Yu, J. Fu, L. Chen et al., "Enhanced thermal conductive property of epoxy composites by low mass fraction of organicinorganic multilayer covalently grafted carbon nanotubes," Composites Science and Technology, vol. 125, pp. 90-99, 2016.

[37] A. Xie, F. Wu, Z. Xu, and M. Wang, "In situ preparation of ultralight three-dimensional polypyrrole/nano $\mathrm{SiO}_{2}$ composite aerogels with enhanced electromagnetic absorption," Composites Science and Technology, vol. 117, pp. 32-38, 2016.

[38] C.-D. Wagner, W.-M. Riggs, L.-E. Davis, J.-E. Moulder, and G.-E. Mulenger, Surface and Interface Analysis, PerkinElmer Corporation, Eden Prairie, Minn, USA, 1979.

[39] W. Zhang, K. Du, C. Yan, and F. Wang, "Preparation and characterization of a novel Si-incorporated ceramic film on pure titanium by plasma electrolytic oxidation," Applied Surface Science, vol. 254, no. 16, pp. 5216-5223, 2008.

[40] H. Machrafi, G. Lebon, and C. S. Iorio, "Effect of volumefraction dependent agglomeration of nanoparticles on the thermal conductivity of nanocomposites: applications to epoxy resins, filled by $\mathrm{SiO}_{2}, \mathrm{AlN}$ and $\mathrm{MgO}$ nanoparticles," Composites Science and Technology, vol. 130, pp. 78-87, 2016. 
[41] C. Chen, H. Wang, Y. Xue et al., "Structure, rheological, thermal conductive and electrical insulating properties of highperformance hybrid epoxy/nanosilica/AgNWs nanocomposites," Composites Science and Technology, vol. 128, pp. 207-214, 2016. 

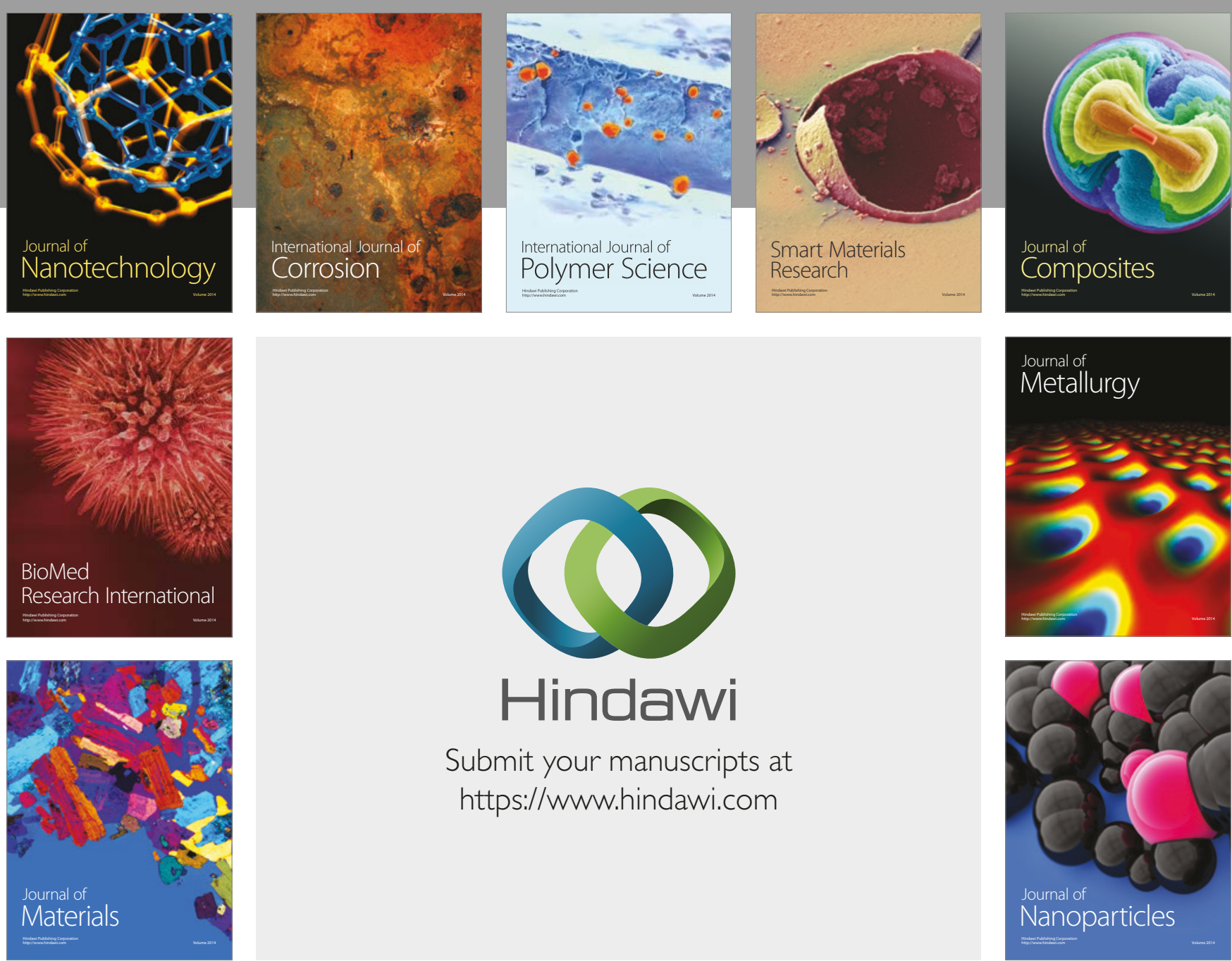

\section{Hindawi}

Submit your manuscripts at

https://www.hindawi.com
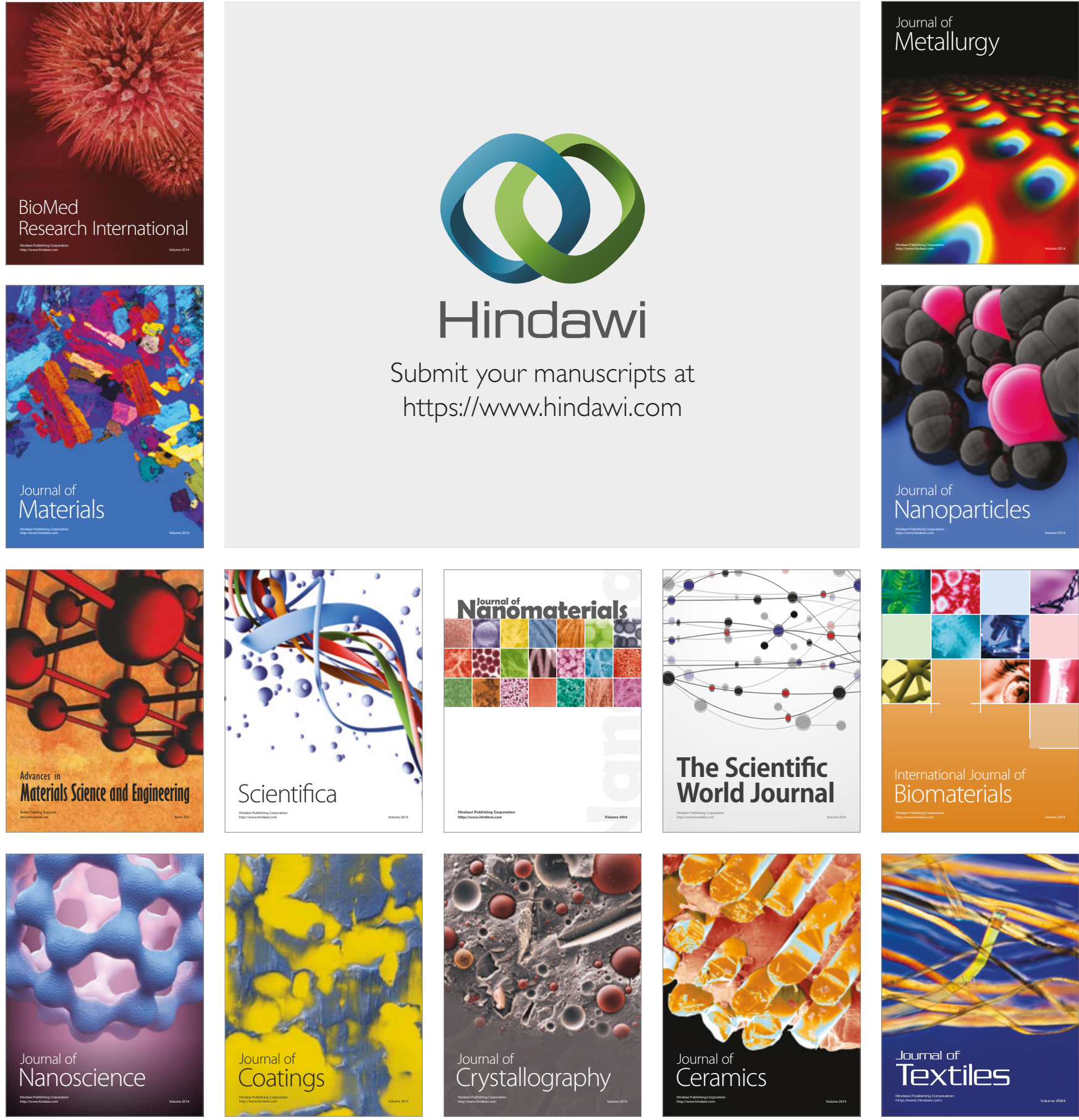

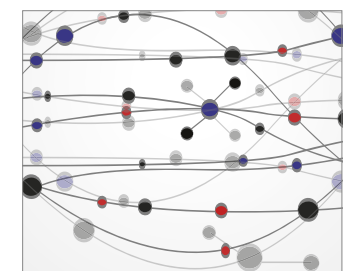

The Scientific World Journal
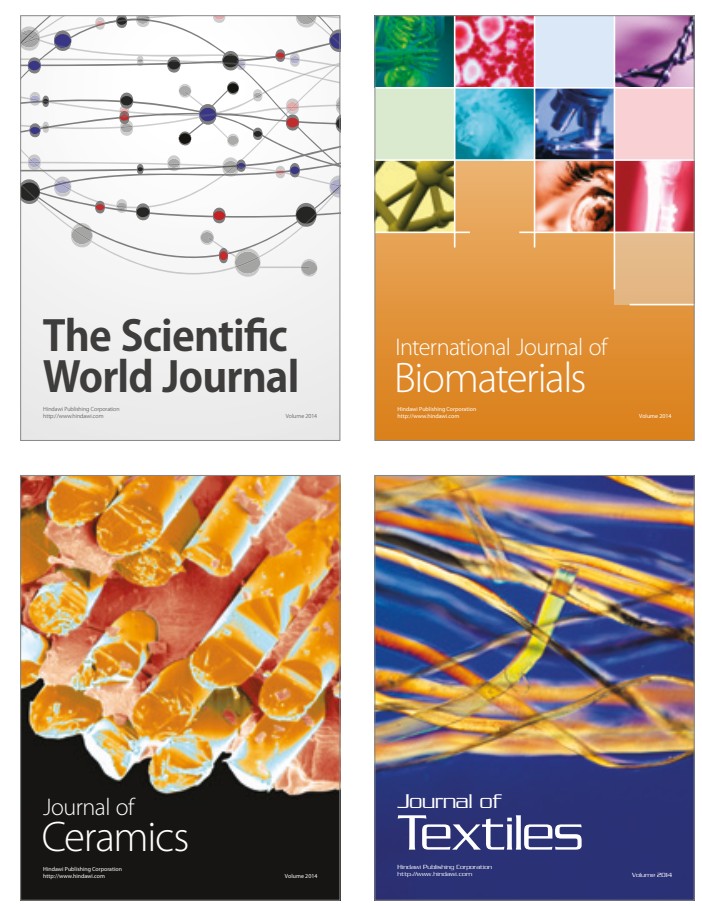